\title{
A Comparative Study of Two Spatial Discretization Schemes for Advection Equation
}

\author{
Huda 0. Bakodah \\ Department of Mathematics, Faculty of Science, Al Faisaliah Campus, King Abdulaziz University, Jeddah, Saudi \\ Arabia \\ Email: hbakodah@kau.edu.sa,h.o.bakodah@gmail.com
}

Received 29 December 2015; accepted 5 March 2016; published 8 March 2016

Copyright @ 2016 by author and Scientific Research Publishing Inc.

This work is licensed under the Creative Commons Attribution International License (CC BY). http://creativecommons.org/licenses/by/4.0/

c) (7) Open Access

\begin{abstract}
In this paper, we describe a comparison of two spatial discretization schemes for the advection equation, namely the first finite difference method and the method of lines. The stability of the methods has been studied by Von Neumann method and with the matrix analysis. The methods are applied to a number of test problems to compare the accuracy and computational efficiency. We show that both discretization techniques approximate correctly solution of advection equation and compare their accuracy and performance.
\end{abstract}

\section{Keywords}

Advection Equation, Finite Difference Method, The Method of Lines, Von Neumann Method

\section{Introduction}

A currently active area of research is the numerical solution of nonlinear partial differential equations and nonlinear integral equations [1]-[7]. An advection equation is fairly in shape but it is one of the most difficult equations to approximate numerically.

The nonlinear advection equation arises in various branches of physics, engineering and applied sciences. The importance of obtaining the exact or approximate solution of this equation is still a significant problem that needs new methods to discover exact or approximate solution.

The linear advection equation is simple in form and yet it is one of the most difficult equations to solve accurately by numerical means [8]. This equation is challenging to solve as it causes some discontinuities with neither dispersion nor dissipation. However, all efforts to use a fixed number of space intervals will result in both 
dispersion and spurious oscillation. The use of traditional symmetric techniques is possible only if the terms of arbitrary second order artificial viscosity or damping are introduced to the equation. Directional (or upwind) methods have shown to be efficient for the purpose of finite difference analyses as it clears the oscillation problem, yet they will not remove it completely. The linear advection equation does provide a good case for testing methods to be used on systems of hyperbolic equations. Many schemes have been tested on it, generally by using propagating step, sine, Gaussian or triangular waveform [9]. These techniques were used to provide a rational for choosing a spatial scheme for first-order hyperbolic equations. The optimal choice is not invariant, but depends on the application.

In this paper, the advection equation is solved by finite difference method [10] [11] and the stability conditions for the scheme are also discussed. Numerical finite difference scheme is developed for obtaining approximate solution to an advection equation using the 3-point formula introduced in [12]. The same problem is considered using modified method of lines [13]-[15], with a new three-point difference [12]. Using this new difference leads to stable schemes for the two methods. Numerical results are shown and compared with analytical solutions.

\section{Finite Difference Method with a Good Spatial Discretization}

Let us consider the equation

$$
\frac{\partial u}{\partial t}+v \frac{\partial u}{\partial x}=0
$$

and $v$ is a nonzero constant velocity, where $u=u(x, t), x \in R$ with the initial condition $u_{0}=u(x, 0)$. The finite difference method begins with discretization the space variable $x$ and the time variable $t$ as follows

$$
t_{j}=t_{0}+j \Delta t, j=0, \cdots, T \text {; and } x_{i}=a+i \Delta x, i=0, \cdots, M, \Delta x=\frac{L}{M} .
$$

The using of some of the finite difference schemes on advection equations can cause unstable solutions. To add stability, upstream (backward or forward) could be used for spatial discretization for the first-order differences. However, for a given spatial accuracy, these differences need to use extra grid points than centered difference. An artificial dissipation (or viscosity) term is normally introduced to a central differencing scheme for stability reasons but it is not easy to determine the magnitude of this term required for the stability and effect of this term on the solutions.

The aim of new method is to develop a good formula with high accuracy for the numerical solution of the advection equation using the spatial discretization presented by Sharaf and Bakodah, [12], using 3-points formula, thus the approximate form of the first derivative is

$$
\begin{aligned}
& \frac{\mathrm{d} u_{1}}{\mathrm{~d} x}=\frac{-3 u_{1}+4 u_{2}-u_{3}}{2 h} \\
& \frac{\mathrm{d} u_{i}}{\mathrm{~d} x}=\frac{-u_{i+1}+u_{i+1}}{2 h}, i=2(1) N-2 \\
& \frac{\mathrm{d} u_{N-1}}{\mathrm{~d} x}=\frac{u_{N-3}-4 u_{N-2}+3 u_{N-1}}{2 h} .
\end{aligned}
$$

Adopting a forward temporal difference scheme, this yields

$$
\begin{aligned}
& u_{1}^{j+1}=\left(1+\frac{3 v k}{2 h}\right) u_{1}^{j}-4 \frac{v k}{2 h} u_{2}^{j}+\frac{v k}{2 h} u_{3}^{j} \\
& u_{i}^{j+1}=\frac{v k}{2 h} u_{i-1}^{j}+u_{i}^{j}-\frac{v k}{2 h} u_{i+1}^{j} \\
& u_{N-1}^{j+1}=\frac{-v k}{2 h} u_{N-3}^{j}+\frac{4 v k}{2 h} u_{N-2}^{j}+\left(1-\frac{3 v k}{2 h}\right) u_{N-1}^{j} .
\end{aligned}
$$

There are two standard methods of the finite-difference equation. In the first method, a finite Fourier series is 
used. In the other method, the equation is expressed in matrix form, and the eigenvalues of the associated matrix are examined. In order to investigate the stability of this scheme by the first method (Von Neumann stability analysis), it is considered

$$
u_{i}^{j}=z_{j} \mathrm{e}^{I k(i h)} .
$$

Replacing $u_{i}^{j}$ in relation (2) from (3), it is obtained:

$$
z_{j+1}=g z_{j}
$$

where

$$
g=\cos (k h)-i \frac{v k}{h} \sin (k h) .
$$

The stability condition $|g|<1$ is fulfilled for all $k$ as long as,

$$
\begin{gathered}
|g|=\sqrt{\cos ^{2}(k h)+\left(\frac{v k}{h}\right)^{2} \sin ^{2}(k h)} \\
|g| \leq 1 \Leftrightarrow\left(\frac{v k}{h}\right)^{2} \leq 1 .
\end{gathered}
$$

That is, the method (2) is stable.

\section{Modified of the Method of Lines}

In the Numerical Method of Lines (NMOL), the partial differential equation (PDE), to be solved, is transformed into a system of ordinary differential equations (ODEs) by discretizing all the independent variables but one [16]. The advection equation, depending on time $t$ and one spatial variable $x$, either $t$ or $x$ can be discretized, and the integration will be carried out along the remaining undiscretized independent variable.

The technique consists of converting the PDE into ODEs either by finite difference spline or by weighted-residual technique, then integrating the resulting ODEs [17]. Finite differencing in the spatial variable led to a set of timedependent ODEs. The advantage of using (NMOL) is that sophisticated software packages exist for the numerical solution of ordinary differential equations. These software packages contain iterative methods for handling non-linearities and feature automatic step-size adjustment and integration order selection to maintain a specified error and to solve the problem with near optimal efficiency. Several recently software packages for automated method of lines solution of arbitrarily defined PDEs have been very successful, particularly for parabolic and elliptic PDE systems.

Such facilities can be improved for hyperbolic equations by incorporating an upwind weighted residual technique. This technique is similar and superior to the use of an artificial viscosity term, and it could be implement easily in any software package. Previous considerations of the (NMOL) to solve PDEs have been geared to parabolic equation and generally used centered, second-order differences. Using these differences on hyperbolic equations can lead to unstable solution. To add stability, upstream (backward or forward) first-order differences could be used for the spatial discretization. But these differences require the use of more grid points than central differences for a given spatial accord. An artificial dissipation (or viscosity) term is often added to a central differencing scheme to add stability but it is difficult to determine the magnitude of this term required for the stability and the effect of this term on the solutions. Other stabilizing techniques that have been employed in the explicit finite difference procedures are generally not applicable to the method of lines approach because they involve manipulation of terms in both the time and space discretization.

In this paper, a modified method of lines using a new three-point difference [12] is used. The use of this new differences leads to stable schemes with good accuracy. In order to apply the method of lines to the advection equation (1), the spatial derivative must be approximated. An equally spaced mesh $x_{i}=i \Delta x$ is used. As in the Ref. [12] a new difference scheme can be used in the calculation of $u_{x}$ 


$$
\begin{aligned}
& \frac{\mathrm{d} u_{1}}{\mathrm{~d} x}=\frac{-3 u_{1}+4 u_{2}-u_{3}}{2 h} \\
& \frac{\mathrm{d} u_{i}}{\mathrm{~d} x}=\frac{-u_{i+1}+u_{i+1}}{2 h}, i=2(1) N-2 \\
& \frac{\mathrm{d} u_{N-1}}{\mathrm{~d} x}=\frac{u_{N-3}-4 u_{N-2}+3 u_{N-1}}{2 h} .
\end{aligned}
$$

It leads to stable schemes with good accuracy. In this section the second method shall be used. The analysis of eigenvalues of the system gives the necessary conditions for the stability of discretization of the problem [18]. The stability corresponds to real and negative values.

By considering equation (1) with the centered difference scheme of order two, then we get

$$
u^{\prime}=A_{1} u
$$

where $A_{1}$ is

$$
A_{1}=\frac{r}{2 h}\left[\begin{array}{ccccccc}
0 & -1 & 0 & 0 & . & . & 0 \\
1 & 0 & -1 & 0 & . & . & 0 \\
0 & 1 & 0 & -1 & . & . & . \\
. & . & . & . & . & . & . \\
. & . & . & . & . & . & -1 \\
0 & . & . & . & . & 1 & 0
\end{array}\right]
$$

and $u=\left[\begin{array}{llll}u_{1} & u_{2} & \cdots & u_{n-1}\end{array}\right]^{\mathrm{T}}$.

Mathematically the difference scheme is stable if there exists a real positive eigenvalues. However, where $A_{1}$ is a tri-diagonal matrix, the corresponding eigenvalue $\lambda_{s}$ of $A$ can be calculated from the relation.

$$
\lambda_{s}=a+2 \sqrt{b c} \cos \frac{s \pi}{n+1}, s=1(1) n
$$

where $a=0, b=-1$ and $c=1$. Thus

$$
\lambda_{s}=2 \sqrt{-1} \cos \frac{s \pi}{n+1}, i=1(1) n
$$

which are pure imaginary values.

So, we consider the non-centered formula approximation

$$
\frac{\partial u}{\partial x}=\frac{1}{2 h}\left[3 u_{i+1}-4 u_{i}+u_{i-1}\right]
$$

with the matrix formula $u^{\prime}=A_{2} u$ where

$$
A_{2}=\left[\begin{array}{cccccccc}
-4 & 3 & 0 & 0 & . & . & . & 0 \\
1 & -4 & 3 & 0 & . & . & . & 0 \\
0 & 1 & -4 & 3 & & & & \\
& & . & . & . & & & \\
& & & . & . & . & & \\
& & & & . & . & . & \\
& & & & & . & . & 3 \\
0 & & & & & & 1 & -4
\end{array}\right] .
$$

Thus the eigenvalues are given by

$$
\lambda_{s}=-4+2 \sqrt{3} \cos \frac{s \pi}{N+1}, \quad s=1(1) N .
$$


These values are real and negative, so the difference scheme is stable.

\section{Numerical Examples}

\subsection{Example 1}

We apply the Finite Difference Method with a good spatial discretization to solve linear advection equation to demonstrate the validity of this method.

Consider the equation

$$
u_{t}(x, t)=u_{x}(x, t)-x^{2} t, x>0, t>0
$$

with the conditions

$$
u(x, 0)=2+\sin x, 0<x \leq 1, u(0, t)=2-\sin t-\frac{1}{12} t^{4} .
$$

With the analytic solution

$$
u(x, t)=2+\sin (x-t)+\frac{1}{12} x^{4}-\frac{1}{3} x^{3} t-\frac{1}{12}(x-t)^{4} .
$$

Using equation (3) we find

$$
u_{i}^{j+1}=\left(\frac{1}{2}-\frac{v k}{2 h}\right) u_{i+1}^{j}+\left(\frac{1}{2}+\frac{v k}{2 h}\right) u_{i-1}^{j}-(i h)^{2}(j k)
$$

let

$$
i=1,2, \cdots, n, \quad j=1,2, \cdots, m, \quad k=0.01, h=0.01, \quad m=5, \quad n=100, \quad v=1 .
$$

Table 1 shows the absolute error $\left|e_{m}\right|=\left|u_{e}-u_{a}\right|$ for the finite difference method in different value of time.

\subsection{Example 2}

We apply the Modified of the Method of lines to solve linear advection equation to demonstrate the validity of this method.

\begin{tabular}{|c|c|c|c|c|c|}
\hline \multirow[b]{2}{*}{$i$} & \multicolumn{5}{|c|}{$\left|u_{\text {exact }}-u_{\text {approximate }}\right|$} \\
\hline & 0.01 & 0.02 & 0.03 & 0.04 & 0.05 \\
\hline 10 & $4.675 \times 10^{-7}$ & 0.000098253 & 0.000277333 & 0.00051992 & 0.000811146 \\
\hline 20 & $1.93417 \times 10^{-6}$ & 0.00039252 & 0.00114473 & 0.00221805 & 0.00357781 \\
\hline 30 & $4.40083 \times 10^{-6}$ & 0.000882787 & 0.00260313 & 0.00510019 & 0.00831948 \\
\hline 40 & $7.8675 \times 10^{-6}$ & 0.00156905 & 0.00465253 & 0.00916632 & 0.0150361 \\
\hline 50 & 0.0000123342 & 0.00245132 & 0.00729293 & 0.0144165 & 0.0237278 \\
\hline 60 & 0.0000178008 & 0.00352959 & 0.0105243 & 0.0208506 & 0.0343945 \\
\hline 70 & 0.0000242675 & 0.00480385 & 0.0143467 & 0.0284687 & 0.0470361 \\
\hline 80 & 0.0000317342 & 0.00627412 & 0.0187601 & 0.0372709 & 0.0616528 \\
\hline 90 & 0.0000402008 & 0.00794039 & 0.0237645 & 0.047257 & 0.0782445 \\
\hline 100 & 0.0000496675 & 0.00980265 & 0.0293599 & 0.0584271 & 0.0968111 \\
\hline
\end{tabular}

Consider the following advection equation

$$
u_{t}+u_{x}=0
$$

with the conditions

Table 1. Absolute errors of the finite difference method, where $t \in\{0.01,0.02,0.03,0.04,0.05\}$. 


$$
\begin{aligned}
& u(x, 0)=\sin \pi x, 0 \leq x \leq 1 \\
& u(0, t)=-\sin \pi t, \quad t \geq 0
\end{aligned}
$$

and the analytic solution

$$
u(x, t)=\sin \pi(x-t)
$$

Substituting in equation (9) we find

$$
\frac{\mathrm{d} u_{i 1}}{\mathrm{~d} t}=-\left(\frac{3 u_{i+1}-4 u_{i}+u_{i-1}}{2 h}\right), \quad i=1,2, \cdots, N
$$

and the condition are

$$
\begin{aligned}
& u_{0}(t)=-\sin \pi t \\
& u_{i}(0)=\sin \pi(i h), \quad i=0, \cdots, N
\end{aligned}
$$

Hence, we can write

$$
\begin{aligned}
\frac{\mathrm{d} u_{1}}{\mathrm{~d} x} & =\frac{-3 u_{1}+4 u_{2}-u_{3}}{2 h} \\
\frac{\mathrm{d} u_{2}}{\mathrm{~d} x} & =\frac{-u_{1}+u_{3}}{2 h} \\
\frac{\mathrm{d} u_{i}}{\mathrm{~d} x} & =\frac{u_{i-2}-4 u_{i-1}+3 u_{i}}{2 h}, \quad i=3(1) N .
\end{aligned}
$$

So, to confirm the accuracy and efficiency of the method, the absolute error $\left|u_{\text {exact }}-u_{\text {approximate }}\right|$ are used (Table 2).

\subsection{Example 3}

Consider the equation

$$
u_{t}+u_{x} u=0
$$

and

$$
u(x, 0)=\sqrt{x}
$$

with the analytic solution

Table 2. Absolute errors of the modified of the method of lines where $N=10$.

\begin{tabular}{cll}
\hline$t$ & $\left|u_{\text {exact }}-u_{\text {approximate }}\right|$ \\
\hline 0.01 & 0.00101545 \\
0.02 & 0.00233093 \\
0.04 & 0.00397634 \\
0.05 & 0.00598185 \\
0.06 & 0.00837779 \\
0.07 & 0.01119470 \\
0.08 & 0.01446333 \\
0.09 & 0.01821444 \\
0.10 & 0.02247890 \\
\hline
\end{tabular}




$$
u(x, t)=0.5\left[-t+\sqrt{t^{2}+4 x}\right] .
$$

In this example we apply the Modified of the Method of lines and the finite difference method to solve linear advection equation to demonstrate the validity of them and compare between them.

Table 3 shows the absolute error $\left|e_{m}\right|=\left|u_{e}-u_{a}\right|$ for the finite difference method equation (2), and the method of lines.

\subsection{Example 4}

Consider the advection equation

with the condition

$$
u_{t}+u u_{x}=0
$$

and the exact solution

$$
u(x, 0)=x
$$

$$
u(x, t)=\frac{x}{1+t} .
$$

This problem is solved for $t=0.1,0.2,0.3, \cdots, 0.8$, with $\Delta x=0.1$.

Table 4 shows the absolute error $\left|e_{m}\right|=\left|u_{e}-u_{a}\right|$.

Table 3. Comparison of the absolute error between finite difference method and the method of lines, for example 1 with $t=0.5$.

\begin{tabular}{ccc}
\hline$x_{i}$ & Finite difference method & The method of lines \\
\hline 0.1 & 0.02067970 & 0.0003747 \\
0.2 & 0.01054830 & 0.0016946 \\
0.3 & 0.00921847 & 0.00400098 \\
0.4 & 0.00851550 & 0.00709063 \\
0.5 & 0.00790683 & 0.0106622 \\
0.6 & 0.00863854 & 0.0144267 \\
0.7 & 0.00712179 & 0.0181591 \\
0.8 & 0.06114460 & 0.0217072 \\
0.9 & 0.07981530 & 0.0249812 \\
1.1 & 0.11121000 & 0.0279372 \\
\hline
\end{tabular}

Table 4. Comparison of the absolute error between finite difference method and the method of lines, for example 2 with $t=0.5$.

\begin{tabular}{cccc}
\hline$x_{i}$ & Finite difference method & The method of lines \\
\hline 0.1 & $\mathbf{0 . 0 0 1 9 6 3 7 7}$ & $2.56842 \times 10^{-11}$ \\
$\mathbf{0 . 2}$ & $\mathbf{0 . 0 0 3 9 2 7 5 5}$ & $3.65622 \times 10^{-11}$ \\
$\mathbf{0 . 3}$ & $\mathbf{0 . 0 0 5 8 9 1 3 2}$ & $4.02893 \times 10^{-11}$ \\
$\mathbf{0 . 4}$ & $\mathbf{0 . 0 0 7 8 5 5 1 0}$ & $4.05249 \times 10^{-11}$ \\
$\mathbf{0 . 5}$ & $\mathbf{0 . 0 0 9 8 1 8 8 7}$ & $3.90807 \times 10^{-11}$ \\
$\mathbf{0 . 6}$ & $\mathbf{0 . 0 1 2 8 3 9 5 0}$ & $3.69000 \times 10^{-11}$ \\
$\mathbf{0 . 7}$ & $\mathbf{0 . 0 0 1 1 9 6 1 5}$ & $\mathbf{0 . 0 8 6 0 8 4 1 0}$ \\
$\mathbf{0 . 8}$ & $\mathbf{0 . 0 9 6 0 7 2 2 0}$ & $3.19589 \times 10^{-11}$ \\
$\mathbf{0 . 9}$ & $\mathbf{0 . 1 2 8 7 9 5 0 0}$ & $2.95723 \times 10^{-11}$ \\
\hline $\mathbf{1 . 0}$ & $2.73389 \times 10^{-11}$ & \\
\hline
\end{tabular}




\section{Conclusions}

From the studied test examples, it has been found that, the modified method of lines gives better results than the finite difference method. Although the modified method of lines is used to approximate the first order hyperbolic differential equation. Thus equations are one of the most difficult classes of PDEs to integrate numerically. To overcome this, a modified MOL scheme is suggested. The results are in good agreement with the exact solution as shown in Table 1, Table 2. The presented method is attractive for hyperbolic, parabolic and elliptic equations.

The methods introduced in this paper for solving the linear and nonlinear advection equation are based on finite difference method. The best choice of the numerical method for a given problem depends on the stability condition.

\section{References}

[1] Biazar, J., Ghazvini, H. and Eslami, M. (2009) He’s Homotopy Perturbation Method for Systems of Integro-Differential Equations. Chaos, Solitons \& Fractals, 39, 1253-1258. http://dx.doi.org/10.1016/j.chaos.2007.06.001

[2] Biazar, J. and Eslami, M. (2011) Modified HPM for Solving Systems of Volterra Integral Equations of the Second Kind. Journal of King Saud University—Science, 23, 35-39. http://dx.doi.org/10.1016/j.jksus.2010.06.004

[3] Biazar, J., Eslami, M. and Islam, M.R. (2012) Differential Transform Method for Special Systems of Integral Equations. Journal of King Saud University—Science, 24, 211-214. http://dx.doi.org/10.1016/j.jksus.2010.08.015

[4] Eslami, M. (2014) New Homotopy Perturbation Method for a Special Kind of Volterra Integral Equations in TwoDimensional Space. Computational Mathematics and Modeling, 25, 135-148. http://dx.doi.org/10.1007/s10598-013-9214-x

[5] Biazar, J. and Eslami, M. (2011) A New Homotopy Perturbation Method for Solving Systems of Partial Differential Equations. Computers \& Mathematics with Applications, 62, 225-234. http://dx.doi.org/10.1016/j.camwa.2011.04.070

[6] Biazar, J., Eslami, M. and Aminikhah, H. (2009) Application of Homotopy Perturbation Method for Systems of Volterra Integral Equations of the First Kind. Chaos, Solitons \& Fractals, 42, 3020-3026. http://dx.doi.org/10.1016/j.chaos.2009.04.016

[7] Biazar, J. and Eslami, M. (2012) A New Method for Solving the Hyperbolic Telegraph Equation. Computational Mathematics and Modeling, 23, 519-527. http://dx.doi.org/10.1007/s10598-012-9153-y

[8] Wazwaz, A.M. (2009) Partial Differential Equations and Solitary Waves Theory. Higher Education Press, Beijing and Springer-Verlag, Berlin Heidelberg. http://dx.doi.org/10.1007/978-3-642-00251-9

[9] George, K. and Twizell, E.H. (2006) Stable Second-Order Finite-Difference Methods for Linear Initial-BoundaryValue Problems. Applied Mathematics Letters, 19, 146-154. http://dx.doi.org/10.1016/j.aml.2005.04.003

[10] Smith, G.D. (1985) Numerical Solution of Partial Differential Equations (Finite Difference Method). 3rd Edition, Oxford University Press, Oxford.

[11] Al-Malki, N.A. and Bakodah, H.O. (2014) A Stable Difference Algorithm for the Solution of Advection Equation. Far East Journal of Applied Mathematics, 88, 139-149.

[12] Sharaf, A.A. and Bakodah, H.O. (2005) A Good Spatial Discretization in the Method of Lines. Applied Mathematics and Computation, 171-172, 1253-1263. http://dx.doi.org/10.1016/j.amc.2005.01.144

[13] Alabdali, F.M. and Bakodah, H.O. (2014) A New Modification of the Method of Lines for First Order Hyperbolic PDEs. Applied Mathematics, 5, 1457-1462. http://dx.doi.org/10.4236/am.2014.510138

[14] Bakodah, H.O. and Banaja, M.A. (2013) The Method of Lines Solution of the Regularized Long-Wave Equation Using Runge-Kutta Time Discretization Method. Mathematical Problems in Engineering, 2013, Article ID: 804317. http://dx.doi.org/10.1155/2013/804317

[15] Banaja, M.A. and Bakodah, H.O. (2015) Runge-Kutta Integration of the Equal Width Wave Equation Using the Method of Lines. Mathematical Problems in Engineering, 2015, Article ID: 274579. http://dx.doi.org/10.1155/2015/274579

[16] Smith, G.D. (1985) Numerical Solution of Partial Differential Equations (Finite Difference Methods). 3rd Edition, Oxford University Press, Oxford.

[17] Schiesser, W.E. (1978) The Numerical Method of Lines, Integration of Partial Differential Equation. 2nd Edition, Clarendon Presses, Oxford.

[18] Carver, M.B. and Hinds, H.W. (1978) The Method of Lines and Advective Equation. Simulation, 31, 59-69. http://dx.doi.org/10.1177/003754977803100205 